University of Nebraska - Lincoln

DigitalCommons@University of Nebraska - Lincoln

Faculty Publications in Computer \& Electronics Electrical \& Computer Engineering, Department Engineering (to 2015)

2007

\title{
A Simple Orthogonal Space-Time-Polarization Block Code
}

\author{
B. J. Wysocki \\ University of Nebraska-Lincoln \\ Tadeusz Wysocki \\ University of Nebraska-Lincoln, wysocki@uow.edu.au \\ J. Seberry \\ University of Wollongong \\ S. Spence Adams \\ Franklin W. Olin College of Engineering \\ H. Sharif \\ University of Nebraska-Lincoln, hsharif@unl.edu
}

Follow this and additional works at: https://digitalcommons.unl.edu/computerelectronicfacpub

Part of the Computer Engineering Commons

Wysocki, B. J.; Wysocki, Tadeusz; Seberry, J.; Adams, S. Spence; and Sharif, H., "A Simple Orthogonal Space-Time-Polarization Block Code" (2007). Faculty Publications in Computer \& Electronics Engineering (to 2015). 34.

https://digitalcommons.unl.edu/computerelectronicfacpub/34

This Article is brought to you for free and open access by the Electrical \& Computer Engineering, Department of at DigitalCommons@University of Nebraska - Lincoln. It has been accepted for inclusion in Faculty Publications in Computer \& Electronics Engineering (to 2015) by an authorized administrator of DigitalCommons@University of Nebraska - Lincoln. 


\title{
A Simple Orthogonal Space-Time-Polarization Block Code
}

\author{
B.J.Wysocki ${ }^{*}$, T.A.Wysocki ${ }^{*}$, Senior Member IEEE, J.Seberry ${ }^{*}$, Senior Member IEEE, S.Spence \\ Adams $^{\dagger}$, Member IEEE, H.Sharif ${ }^{\dagger}$, Senior Member IEEE
}

\begin{abstract}
This letter proposes a simple but highly efficient technique to jointly utilize space, time, and polarization diversities. The technique is based on extending orthogonal space-time block codes into the quaternion domain and utilizing a description of the dual-polarized signal by means of quaternions, which are a generalization of complex numbers. In the given example, the achievable performance gain for two transmit and one receive antennas is approximately $6 \mathrm{~dB}$ at a bit error rate of $10^{-4}$ when compared with the Alamouti code.
\end{abstract}

\section{INTRODUCTION}

The mechanism of a diversity gain for orthogonal space-time block codes (OSTBCs) relies on providing extra transmission channels that experience independent Rayleigh fading through the use of additional transmit antennas [1]. However, it has been proven that the usage of more than 2 transmission antennas requires either a reduction in the code rate for complex signal constellations [2] or a significant increase in receiver complexity due to the loss of orthogonality and, in general, no means of a decoupled maximum likelihood (ML) decoding method [3].

Recently, the demand for high rates in mobile communications has raised interest in applying polarization diversity, often together with other forms of diversity [4,5]. Polarization diversity is a technique where information is transmitted and received simultaneously on orthogonally polarized waves with fade-independent propagation characteristics. It has been shown $[4,5]$ that it can significantly add to the performance improvements offered by other diversity techniques, and be nearly as effective as spatial diversity for base station antennas, without a noticeable increase in their dimensions.

* B.J.Wysocki, T.A.Wysocki and J.Seberry are with the Faculty of Informatics, University of Wollongong, New South Wales, NSW 2522, Australia (e-mail: bjw, wysocki, jennie@uow.edu.au).

$\rightarrow$ S.Spence Adams is with Franklin W. Olin College of Engineering, Olin Center, Olin Way, Needham, MA 02492-1200, (e-mail: sarah.adams@olin.edu).

* H.Sharif is with the Computer and Electronics Engineering Department at the University of Nebraska-Lincoln in the Peter Kiewit Institute, (email: hsharif@mail.unomaha.edu).
Some efforts to utilize polarization diversity jointly with OSTBCs have been previously described [6], but these were limited to the use of conventional complex OSTBCs without attempting to introduce a code specially designed to jointly utilize all three diversity techniques. In this letter, we propose to design orthogonal space-time-polarization block codes (OSTPBCs) based on recently defined orthogonal designs with quaternion elements [7]. Quaternions were used in the past to construct complex OSTBCs, e.g. [8] but the technique presented in this paper is significantly different, as our approach does not involve non-linear processing as the one presented in [8]. It should be noted that the similar performance gains can be achieved using conventional OSTBCs but at the expense of significant increase in the receiver dimensions due to antenna spacing requirements.

\section{QuATERnionic Representation of Dual Polarized SIGNALS}

Quaternions, invented by Hamilton in 1843, are very well suited to describe rotations and sequences of rotations, and were used by Isayeva and Sarytchev [9] to describe dualpolarized radio signals, whereby two complex signals are rotated one against another by 90 degrees on the polarization plane. Using that approach, two complex signals

$$
z_{1}=x_{1}+y_{1} i \text { and } z_{2}=x_{2}+y_{2} i
$$

being orthogonal to each other on a polarization plane form a quaternion number $s=z_{1}+z_{2} j=x_{1}+y_{1} i+x_{2} j+y_{2} k$ where $i, j$, and $k$ satisfy $i^{2}=j^{2}=k^{2}=-1$ and $i j k=-1$ and $x_{1}, y_{1}$, $x_{2}, y_{2}$ are real numbers. We note that multiplication of quaternion numbers is non-commutative and for a complex variable $z$, we have $z j=j z^{*}$, where $z^{*}$ is the complex conjugate of $z$.

For a quaternion variable $s$, the quaternion conjugate $s^{Q}$ is defined analogously to the complex conjugate, i.e.

$$
\begin{aligned}
s^{Q} & =\left(x_{1}+y_{1} i+x_{2} j+y_{2} k\right)^{Q} \\
& =x_{1}-y_{1} i-x_{2} j-y_{2} k
\end{aligned}
$$

which implies that $s^{Q} s=|s|^{2}=\left|z_{1}\right|^{2}+\left|z_{2}\right|^{2}=x_{1}{ }^{2}+y_{1}{ }^{2}+x_{2}{ }^{2}+y_{2}{ }^{2}$. For matrices of quaternion numbers or variables, the quaternion transform is analogous to the Hermitian transform 
for complex matrices: For a quaternion matrix $\mathbf{S}=\left[s_{l m}\right], \mathbf{S}^{Q}=$ $\left[s^{Q}{ }_{m l}\right]$.

A rotation $s_{\varphi}$ of the signal $s$ on a polarization plane by an angle of $\varphi$ can be simply represented in the quaternion notation as:

$s_{\varphi}=s e^{j \varphi}=\left(z_{1}+z_{2} j\right) e^{j \varphi}=\left(z_{1}+z_{2} j\right)[\cos (\varphi)+j \sin (\varphi)]$

Similarly, any change to the polarization bases, e.g. due to differences between transmit and receive antenna alignments, can be easily represented in the quaternion notation, as long as the polarization bases are orthogonal [9].

When both transmitter and receiver use dual-polarized antennas that can separately transmit/receive signals in two orthogonal polarizations, the channel between transmitter and receiver is described by a channel gain matrix $\mathbf{H}$, given by:

$$
\mathbf{H}=\left[\begin{array}{ll}
h_{11} & h_{12} \\
h_{21} & h_{22}
\end{array}\right],
$$

where $h_{11}$ and $h_{22}$ are complex channel gains for signals received with the same polarization as they were transmitted, and $h_{12}$ and $h_{21}$ are complex channel gains for a cross-polar scatter, i.e. signals received with different polarization from that at which it was transmitted due to scatter, reflections and polarization twist between the transmit and receive antennas.

Introducing representation of a quaternion variable $s=z_{1}+$ $z_{2} j$ as $s=\left[z_{1}, z_{2}\right]$, transmission of the dual-polarized signal through the channel described by a matrix $\mathbf{H}$ can be modeled as a product $s \mathbf{H}$. Hence the received quaternion signal $r$ is given by:

$$
\begin{aligned}
r & =s \mathbf{H}+n \\
& =\left[z_{1}, z_{2}\right] \mathbf{H}+\left[n_{1}, n_{2}\right] \\
& =\left[z_{1} h_{11}+z_{2} h_{21}, z_{1} h_{12}+z_{2} h_{22}\right]+\left[n_{1}, n_{2}\right] \\
& =\left(z_{1} h_{11}+z_{2} h_{21}\right)+\left(z_{1} h_{12}+z_{2} h_{22}\right) j+n_{1}+n_{2} j
\end{aligned}
$$

where $n_{1}, n_{2}$ are complex additive noises being the independent identically distributed (i.i.d.) zero-mean two dimensional Gaussian random variables with identical variance per dimension.

\section{Orthogonal Space-Time-Polarization Block Codes}

Orthogonal space-time block codes (OSTBCs) have been proposed to jointly utilize space and time diversities and allow for a decoupled maximum likelihood (ML) decoding at the receiver [10]. To jointly utilize space, time, and polarization diversities, we propose here orthogonal space-timepolarization block codes (OSTPBCs). Such codes can be derived from quaternion orthogonal designs, which have recently been defined [7]. A quaternion orthogonal design (QOD) of order $n$ on the quaternion variables $s_{1}, s_{2}, \ldots, s_{u}$ is an $n \times n$ matrix $\mathbf{D}$ with entries from the set $\left\{0, \pm q s_{m}, \pm q s_{m}^{Q} ; 1 \leq m \leq u, q \in\{1, i, j, k\}\right\}$, satisfying

$$
\mathbf{D}^{Q} \mathbf{D}=\left(\sum_{l=1}^{u} \lambda_{l}\left|s_{l}\right|^{2}\right) \mathbf{I}_{n},
$$

where $\mathbf{I}_{\mathrm{n}}$ is the $n \times n$ identity matrix and $\lambda_{l}$ is the number of times the variable $s_{l}$ appears in each column.

Some techniques that can be used to construct QODs have been recently presented [7]. Consider the following simple OSTPBC, derived from a QOD of order 2, for two transmit dual-polarized antennas:

$$
\mathbf{Q}_{1}=\left[\begin{array}{cc}
z_{1}+z_{2} j & z_{2}+z_{1} j \\
-z_{2}^{*}+z_{1}^{*} j & z_{1}^{*}-z_{2}^{*} j
\end{array}\right]
$$

It can be easily noticed that $\mathbf{Q}_{1}$ comprises two Alamouti [11] OSTBCs, one used for one polarization and another one, with switched columns, used for orthogonal polarization. Using the same notation as in (5), $\mathbf{Q}_{1}$ can be expressed as:

$$
\mathbf{Q}_{1}=\left[\begin{array}{cccc}
z_{1} & z_{2} & z_{2} & z_{1} \\
-z_{2}^{*} & z_{1}^{*} & z_{1}^{*} & -z_{2}^{*}
\end{array}\right]
$$

where odd columns represent signals transmitted through one polarization and even columns represent signals transmitted through the polarization orthogonal to the first one.

For a single receive dual-polarized antenna, the channels between dual-polarized transmit antennas Tx1 and Tx2, and the dual-polarized receive antenna Rx1 are described by their own channel gain matrices, $\mathbf{H}^{(1)}=\left[h_{m n}^{(1)}\right]_{2 \times 2}$ and $\mathbf{H}^{(2)}=\left[h_{m n}^{(2)}\right]_{2 \times 2}$, respectively (see Fig. 1). The received signal vector $\mathbf{R}$ is given by

$$
\begin{aligned}
\mathbf{R} & =\mathbf{Q}_{1}\left[\begin{array}{l}
\mathbf{H}^{(1)} \\
\mathbf{H}^{(2)}
\end{array}\right]+\left[\begin{array}{ll}
n_{11} & n_{12} \\
n_{21} & n_{22}
\end{array}\right] \\
& =\left[\begin{array}{c}
z_{1} h_{11}^{(1)}+z_{2} h_{21}^{(1)}+z_{2} h_{11}^{(2)}+z_{1} h_{21}^{(2)} \\
-z_{2}^{*} h_{11}^{(1)}+z_{1}^{*} h_{21}^{(1)}+z_{1}^{*} h_{11}^{(2)}-z_{2}^{*} h_{21}^{(2)}
\end{array}\right], \\
& +\left[\begin{array}{c}
z_{1} h_{12}^{(1)}+z_{2} h_{22}^{(1)}+z_{2} h_{12}^{(2)}+z_{1} h_{22}^{(2)} \\
-z_{2}^{*} h_{12}^{(1)}+z_{1}^{*} h_{22}^{(1)}+z_{1}^{*} h_{12}^{(2)}-z_{2}^{*} h_{22}^{(2)}
\end{array}\right] j+\left[\begin{array}{l}
n_{11} \\
n_{21}
\end{array}\right]+\left[\begin{array}{l}
n_{12} \\
n_{22}
\end{array}\right] j
\end{aligned}
$$

where $n_{m l} ; m, l=1,2$ represent complex noises being the independent identically distributed (i.i.d.) zero-mean two dimensional Gaussian random variables with identical variance per dimension.

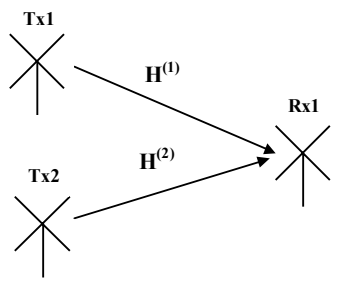

Fig. 1. Considered scenario of 2 transmit (Tx) and one receive ( $\mathrm{Rx})$ dualpolarized antennas.

Assuming perfect channel knowledge at the receiver, i.e. assuming that matrices $\mathbf{H}^{(1)}$ and $\mathbf{H}^{(2)}$ are known and constant for some reasonable time, as in the case of quasi static conditions, the ML decoding means finding a pair of elements $z_{1}$ and $z_{2}$ of the complex signal constellation $\mathbf{Z}$ that minimizes the following metric: 


$$
\begin{aligned}
\left\|\mathbf{R}-\mathbf{Q}_{1}\left[\begin{array}{c}
\mathbf{H}^{(1)} \\
\mathbf{H}^{(2)}
\end{array}\right]\right\| & =r_{1}-z_{1} h_{11}^{(1)}-z_{2} h_{21}^{(1)}-z_{2} h_{11}^{(2)}-z_{1} h_{21}^{(2)} \\
& -z_{1} h_{12}^{(1)} j-z_{2} h_{22}^{(1)} j-z_{2} h_{12}^{(2)} j-\left.z_{1} h_{22}^{(2)} j\right|^{2}, \\
& +\mid r_{2}+z_{2}^{*} h_{11}^{(1)}-z_{1}^{*} h_{21}^{(1)}-z_{1}^{*} h_{11}^{(2)}+z_{2}^{*} h_{21}^{(2)} \\
& +z_{2}^{*} h_{12}^{(1)} j-z_{1}^{*} h_{22}^{(1)} j-z_{1}^{*} h_{12}^{(2)} j+\left.z_{2}^{*} h_{22}^{(2)} j\right|^{2}
\end{aligned}
$$

where $r_{1}$ and $r_{2}$ are the elements of the receive vector $\mathbf{R}$.

Utilizing the principles of quaternionic arithmetic given in Section II, the decoding rule can be simplified and proven to be decoupled. The resulting decoding statistics for $z_{1}$ is:

$$
\begin{aligned}
\min _{z_{1} \in Z} & \left|z_{1}\right|^{2}\left(\left|g_{1}\right|^{2}+\left|g_{2}\right|^{2}+\left|g_{3}\right|^{2}+\left|g_{4}\right|^{2}\right) \\
& -2 \operatorname{Re}\left\{r_{1}^{O} z_{1}\left(g_{1}+g_{2} j\right)+r_{2}^{O} z_{1}^{*}\left(g_{3}+g_{4} j\right)\right\}
\end{aligned}
$$

and for $z_{2}$ it is:

$$
\begin{aligned}
\min _{z_{2} \in Z} \mid & \left.z_{2}\right|^{2}\left(\left|g_{1}\right|^{2}+\left|g_{2}\right|^{2}+\left|g_{3}\right|^{2}+\left|g_{4}\right|^{2}\right) \\
& -2 \operatorname{Re}\left\{r_{1}^{Q} z_{2}\left(g_{3}+g_{4} j\right)-r_{2}^{Q} z_{2}^{*}\left(g_{1}+g_{2} j\right)\right\}
\end{aligned}
$$

where

$$
\begin{array}{ll}
g_{1}=h_{11}^{(1)}+h_{21}^{(2)}, & g_{2}=h_{12}^{(1)}+h_{22}^{(2),}, \\
g_{3}=h_{21}^{(1)}+h_{11}^{(2)}, & g_{4}=h_{22}^{(1)}+h_{12}^{(2)}
\end{array}
$$

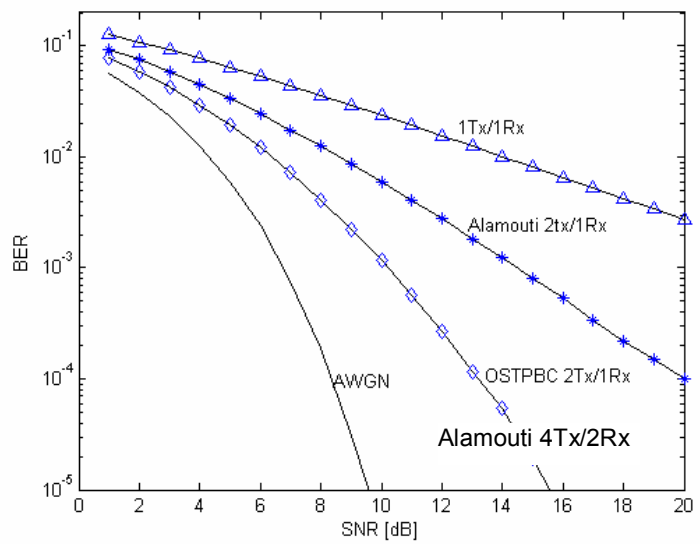

Fig. 2. Bit-error-rate (BER) performance of the developed scheme (OSTPBC) combined with QPSK modulation in a slow flat Rayleigh fading channel experiencing random cross-polar scatter compared with the Alamouti's scheme and a single transmit/single receive antenna system.

\section{Simulation Results}

To assess performance gain that can be achieved using the developed OSTPBC for 2 transmit and a single receive dualpolarized antennas, the system was implemented using MATLAB. The following conditions were assumed:

- The QPSK $\{\pi / 4,3 \pi / 4,5 \pi / 4,7 \pi / 4\}$ signal constellation was applied, and the code based on $\mathbf{Q}_{1}$ was used (OSTPBC).

- Total transmitted power in both polarizations and through both antennas was equal to 1 , and equally distributed per antenna and per polarization.
- Channel coefficient matrices, $\mathbf{H}^{(1)}$ and $\mathbf{H}^{(2)}$ were assumed known at the receiver and kept constant for 1024 data bits.

- In the dynamic indoor scenario, variances of channel coefficients can change randomly due to changing scattering conditions. Hence we only assumed that the sum of variances of all the coefficients was equal to 1 and that the variance of real and imaginary part of a particular coefficient was identical. The variances were then drawn randomly, every time the new set of coefficients was drawn.

- The channel coefficients were generated as random complex Gaussian i.i.d. variables.

- The additive noise was assumed to be AWGN added uniformly for each polarization and each real/imaginary component (a quaternion zero-mean Gaussian variable).

To compare the performance achieved, we also simulated a single polarization, single transmit - single receive system experiencing slow Rayleigh fading, and a single polarization Alamouti systems with two transmit and one receive $(2 \mathrm{Tx} / 1 \mathrm{Rx})$ antennas and with $4 \mathrm{Tx} / 2 \mathrm{Rx}$ configuration [11]. The simulation parameters were adjusted for those systems to ensure consistency of the conditions. The simulation results are presented in Fig. 2, where we have also drawn the curve representing performance of QPSK in the AWGN channel. It is clearly visible that the proposed OSTPBC performs very robustly in a realistic indoor environment and significantly outperforms the $2 \mathrm{Tx} / 1 \mathrm{Rx}$ Alamouti system and performs exactly the same as $4 \mathrm{Tx} / 2 \mathrm{Rx}$ Alamouti scheme. This is due to the fact that the proposed OSTPBC is equivalent to the $4 \mathrm{Tx} / 2 \mathrm{Rx}$ Alamouti scheme. The benefit of the proposed scheme follows from the fact that its physical dimensions are similar to the system using $2 \mathrm{Tx} / 1 \mathrm{Rx}$ Alamouti OSTBC with the performance of the $4 \mathrm{Tx} / 2 \mathrm{Rx}$ code. For a fair comparison, the average total received power in all systems before adding noise was set to 1 , and we used the same SNR normalization as used in [11].

In addition, the $2 \mathrm{Tx} / 1 \mathrm{Rx}$ Alamouti scheme is susceptible to the cross-polar scatter unless circularly polarized antennas are used with the dimensions fully comparable to antenna system of the proposed scheme.

\section{CONClusions}

In this letter, we proposed a method to jointly utilize space, time, and polarization diversities by introducing the concept of orthogonal space-time-polarization block codes (OSTPBCs). The given example performs very well in an environment characterized by slow Rayleigh fading combined with slowly changing cross-polar scatter. The use of quaternionic arithmetic to design OSTPBCs and to perform decoding opens a new area for increasing the total diversity gain and improving system performance in harsh radio environments. In the paper, we considered a very simple channel model. More realistic channel models taking to account possibility of correlation between polarizations need to be considered as 
well. Future research efforts will be devoted to designing maximum rate, full diversity OSTPBCs for two and more transmit antennas.

\section{REFERENCES}

[1] G.J.Foschini, and M.J.Gans: "On limits of wireless communication in fading environment when using multiple antennas," Wireless Personal Communications, Vol.6, no.3, pp.311-335, 1998.

[2] X.-B.Liang, and X.G. Xia: "On the nonexistence of rate-one generalized complex orthogonal designs", IEEE Trans. Information Theory, Vol.49, no.11, pp.2984-2988, 2003.

[3] L.A. Dalton, and C.N. Georghiades: "A full-rate, full-diversity fourantenna quasi-orthogonal space-time block code," IEEE Trans. Wireless Commun., Vol. 4, No. 2, pp. 363-366, 2005.

[4] C.B.Dietrich, K.Dietze, J.R.Nealy, and W.L.Stutzman: "Spatial, polarization, and pattern diversity for wireless handheld terminals," IEEE Trans. Antennas Propagat., Vol. 49, pp.1271 - 1281, 2001.

[5] R.U.Nabar, H.Bölcskei, V.Erceg, D.Gesbert, A.J.Paulraj: "Performance of multiantenna signalling techniques in the presence of polarization diversity," IEEE Trans. Signal Processing, Vol. 50, pp. 2553-2562, 2002.

[6] N.M.Murad, D.Carsenat, and B.Jecko: "Space-polarization diversity for a $2 \times 2$ MIMO using space time block codes," IEEE/ACES Int. Conf. Wireless Communications and Applied Computational Electromagnetics, 3-7 April 2005, pp.482-485.

[7] J.Seberry, K.Finlayson, S. Spence Adams, T.A Wysocki, and T.Xia: "The theory of orthogonal designs over the quaternion domain" - under review.

[8] A.R. Calderbank, S. Das, N. Al-Dhahir, and S.N. Diggavi: "A Novel Full-Rate Full-Diversity Orthogonal STBC with Application to WiMAX“, IEEE VTC Fall 2005, CDROM.

[9] O.M.Isaeva, and V.A.Sarytchev: "Quaternion presentations polarization state," Proc. $2^{\text {nd }}$ IEEE Topical Symposium of Combined OpticalMicrowave Earth and Atmosphere Sensing, Atlanta, GA USA, 3-6 April 1995, pp.195-196.

[10] V.Tarokh, H.Jafarkhani, A.R.Calderbank: "Space-time block codes from orthogonal designs," IEEE Trans. on Info. Th, Vol. 45, No. 5, pp. 1456$1467,1999$.

[11] S. Alamouti: "A simple transmit diversity technique for wireless communications," IEEE J. on Selected Areas in Communications, Vol. 16, No. 8, pp. 1451-1458, 1998. 